This item was submitted to Loughborough's Research Repository by the author.

Items in Figshare are protected by copyright, with all rights reserved, unless otherwise indicated.

\title{
To stop or not to stop: contrasting compliant and non-compliant driver behaviour at rural rail level crossings
}

PLEASE CITE THE PUBLISHED VERSION

https://doi.org/10.1016/j.aap.2017.09.004

\section{PUBLISHER}

(C) Elsevier

VERSION

AM (Accepted Manuscript)

\section{PUBLISHER STATEMENT}

This work is made available according to the conditions of the Creative Commons Attribution-NonCommercialNoDerivatives 4.0 International (CC BY-NC-ND 4.0) licence. Full details of this licence are available at: https://creativecommons.org/licenses/by-nc-nd/4.0/

\section{LICENCE}

CC BY-NC-ND 4.0

\section{REPOSITORY RECORD}

Beanland, Vanessa, Paul M. Salmon, Ashleigh Filtness, Michael G. Lenne, and Neville Stanton. 2019. "To Stop or Not to Stop: Contrasting Compliant and Non-compliant Driver Behaviour at Rural Rail Level Crossings". figshare. https://hdl.handle.net/2134/28392. 
To stop or not to stop: Contrasting compliant and non-compliant driver behaviour at rural rail level crossings

\author{
Vanessa Beanland ${ }^{1,2 *}$, Paul M. Salmon ${ }^{1,2}$, Ashleigh J. Filtness ${ }^{2,3}$, Michael G. Lenné ${ }^{2}$ and
} Neville A. Stanton ${ }^{1,4}$

${ }^{1}$ Centre for Human Factors and Sociotechnical Systems, University of the Sunshine Coast, Sippy Downs, QLD, Australia

${ }^{2}$ Monash University Accident Research Centre, Monash University, Clayton, VIC, Australia

${ }^{3}$ Loughborough Design School, Loughborough University, Loughborough, United Kingdom

${ }^{4}$ Transportation Research Group, Civil, Maritime, Environmental Engineering \& Science Unit, University of Southampton, Southampton, United Kingdom

*Corresponding author. vbeanlan@usc.edu.au

This is an author-accepted version of a published paper. Citation details for the published version are:

Beanland, V., Salmon, P. M., Filtness, A. J., Lenné, M. G., \& Stanton, N. A. (2017). To stop or not to stop: Contrasting compliant and non-compliant driver behaviour at rural rail level crossings. Accident Analysis and Prevention, 108C, 209-219. doi: 10.1016/j.aap.2017.09.004 


\begin{abstract}
Many rail level crossings (RLXs) have only passive protection, such as static signs instructing road users to stop, yield, or look for trains. Stop signs have been suggested as a low-cost option to improve safety at passive RLXs, as requiring drivers to stop should encourage safe behaviour. However, field observations have noted high rates of non-compliance at stop-controlled RLXs. To explore this further, we conducted an on-road study to identify factors that influence compliance at stop-controlled RLXs. Twenty-two drivers drove a $30.5 \mathrm{~km}$ route in rural Australia, encompassing three stop-controlled RLXs. In over half of all cases (59\%) drivers stopped completely at the RLX; on $27 \%$ of crossings drivers executed a rolling stop, and on $14 \%$ of crossings drivers violated the stop controls. Rolling stops were defined as a continuous deceleration to $<10 \mathrm{~km} / \mathrm{h}$, but remaining above $0 \mathrm{~km} / \mathrm{h}$, before accelerating to $>10 \mathrm{~km} / \mathrm{h}$. Behavioural patterns, including visual checks and decision-making, were similar when comparing drivers who made complete versus rolling stops. Non-compliant drivers did not differ from compliant drivers in approach speeds, but spent less time visually checking for trains. Post-drive interviews revealed some drivers wilfully disregarded the stop sign, whereas others did not notice the stop sign. Those who intentionally violated noted trains were infrequent and suggested sight distance was good enough (even though all crossings had been formally assessed as having inadequate sight distance). Overall the results suggest most drivers exhibit safe behaviour at passive RLXs, but a notable minority disregard or fail to notice signs. Potential avenues for redesigning passive RLXs to improve safety are discussed.
\end{abstract}

Keywords: rail level crossings; passive warnings; stop signs; rail road crossing 


\section{Introduction}

Safety issues are prevalent at rail level crossings (RLXs) worldwide (Evans, 2011; Mulvihill et al., 2016; Pyrgidis et al., 2016). Accordingly, a range of active and passive traffic control devices are used to guide road user decision-making at RLXs. Active RLXs incorporate warnings (e.g., flashing lights, bells) and/or barriers that activate when a train is approaching, whereas passive RLXs have only static signs and road markings. Crash analyses indicate that active RLXs, especially those with barriers or gates, have superior safety performance (Austin and Carson, 2002; McCollister and Pflaum, 2007; Raub, 2009; Saccomanno et al., 2007). However, passive RLXs predominate in many countries, constituting approximately two-thirds of Australia's public RLXs (ATSB, 2008). Although upgrading to active controls is a government priority it remains cost-prohibitive, with a low benefit-cost ratio (Cairney et al., 2002). This has prompted researchers and practitioners to seek cost-effective ways of improving safety at passive RLXs (Read et al., 2017).

One low-cost option could be passive signs that promote safer decision-making. The prototypical sign at passive RLXs is a crossbuck, sometimes with the instruction to yield, slow or look for trains (Yeh and Multer, 2007). These configurations place the onus on individuals to decide whether to stop. An alternative is to add stop signs, which should reduce decision uncertainty by legally obliging road users to stop. At stop-controlled RLXs, it is common to have a stop sign placed immediately at the RLX, usually with a crossbuck to indicate the presence of an RLX. Some stop-controlled RLXs include additional signage (e.g., advance warning signs that signal an upcoming passive RLX and/or the need to stop ahead) and road markings, such as a painted stop line (see Figure 1).

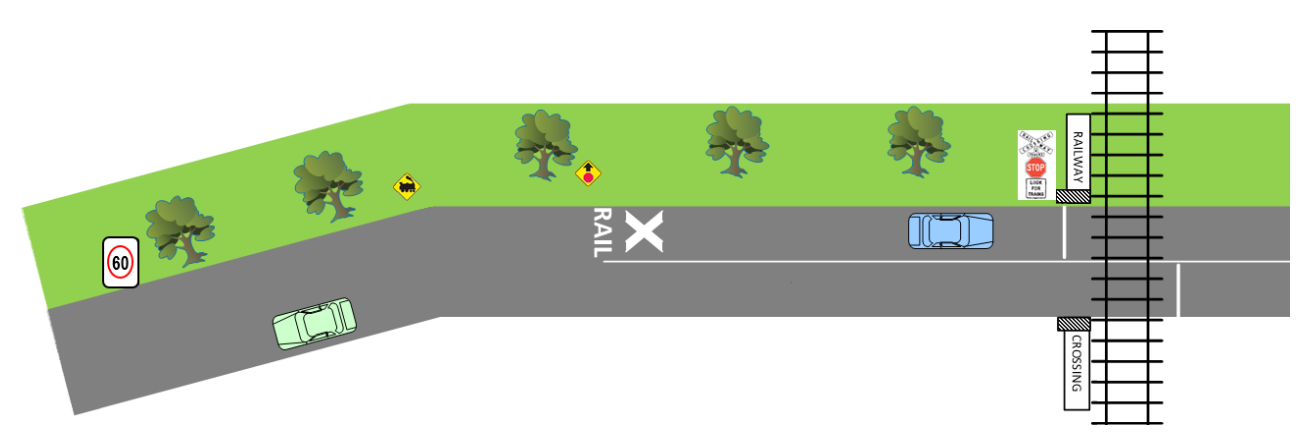

Figure 1. Example configuration for a stop-controlled rail level crossing in Australia. Signs at the crossing include a crossbuck, stop sign, a sign that says "LOOK FOR TRAINs" and signs flanking the road saying "RAILWAY" and "CROSSING". Signs on approach include an icon of a steam train (indicating a passive rail level crossing ahead) and a sign indicating a stop sign ahead. 


\subsection{Driver compliance at stop-controlled $R L X s$}

Stop controls theoretically encourage all drivers to make safe, consistent decisions at passive RLXs (Ward and Wilde, 1996). Supporting this, a recent simulator study found that stop signs increased both stopping and looking behaviour at passive RLXs (Liu et al., 2016). Despite this, there is evidence that many drivers violate RLX stop signs (Kasalica et al., 2012; Lenné et al., 2011; Rudin-Brown et al., 2012; Russell et al., 2007; Tey et al., 2011, 2013). As summarised in Table 1, compliance rates vary greatly, with field observations in USA, Australia and Serbia revealing real-world compliance rates from 12\% and 43\% (Kasalica et al., 2012; Russell et al., 2007; Tey et al., 2011).

Compliance at stop-controlled RLXs tends to be higher in simulator studies, ranging from $44 \%$ to 74\% (Lenné et al., 2011; Rudin-Brown et al., 2012; Tey et al., 2013), but is still well below the compliance rates for active controls: the same simulator studies found $76-80 \%$ compliance at traffic lights (Lenné et al., 2011; Rudin-Brown et al., 2012) and 96-100\% compliance with flashing lights (Lenné et al., 2011; Tey et al., 2013). Two simulator studies incorporated interviews that explored drivers' interpretations of traffic controls. Strikingly, 29\% of participants in Queensland, Australia, and 88\% of participants in Victorian, Australia, suggested the correct response at a stop-controlled RLX is to "look for a train" and/or "slow down" (Rudin-Brown et al., 2012; Tey et al., 2013).

Table 1

Summary of previous research findings on compliance at stop-controlled rail level crossings.

\begin{tabular}{|l|l|l|c|}
\hline Study & Location & Study type & Compliance \\
\hline Russell et al. (2007) & Kansas, USA & Field observation & $12 \%$ \\
\hline Tey et al. (2011) & Queensland, Australia & Field observation & $41 \%$ \\
\hline Kasalica et al. (2012) & Căpljinac, Serbia & Field observation & $43 \%^{+}$ \\
\hline Rudin-Brown et al. (2012) & Victoria, Australia & Simulator & $44 \%^{+}$ \\
\hline Lenné et al. (2011) & Victoria, Australia & Simulator & $60 \%^{+}$ \\
\hline Tey et al. (2013) & Queensland, Australia & Simulator & $67-74 \%$ \\
\hline
\end{tabular}

${ }^{\dagger}$ Denotes studies that only collected data when a train was approaching, so non-compliant drivers crossed in front of an oncoming train. All other studies represent a mix of train-present and train-absent encounters.

\subsection{Reasons for noncompliance}

A key limitation is that previous research has not identified the reasons underlying noncompliance. Although some simulator studies have explored this, there remains limited understanding of why some drivers disregard stop signs at RLXs. This is particularly so for RLX 
encounters in naturalistic settings. This is a significant knowledge gap, as understanding the factors driving non-compliance should inform the design of interventions to prevent it.

Researchers have suggested that noncompliance in US studies stems from "indiscriminate application" of stop signs at RLXs (Raub, 2009; Russell et al., 2007). US regulations permit installation of stop signs at any RLX with at least two trains per day. This means stop signs are used at very low-volume RLXs, whereas elsewhere in the road network they are installed at relatively busy non-RLX intersections (Raub, 2009). The consequence is that whereas drivers often encounter traffic at road intersections with stop signs, they rarely encounter trains at stop-controlled RLXs, meaning the RLX environment deviates from the prototypical stop-controlled intersection.

Other jurisdictions have more stringent regulations regarding the use of stop controls. In Australia, stop signs are only installed at RLXs with restricted visibility on approach (Standards Australia, 2007). Specifically, stop signs must be used at passive crossings if approaching road users would not have enough time to stop safely from the point at which they can first see far enough down the tracks to detect an oncoming train. Ideally, such strict regulation should foster greater compliance; however, this presupposes that drivers recognise and agree with the reasons for installing stop controls. This is questionable given that Australian research found many drivers declare "slowing down" is the appropriate response to a stop sign (Rudin-Brown et al., 2012; Tey et al., 2013).

One avenue for understanding drivers' behaviour is through Neisser's (1976) perceptual cycle model, which posits that our perception of the world is a constructive, iterative process that originates from our schemata, or mental models of what we expect to encounter. The content of our schemata is based on our past knowledge and experience, and guide our exploration or information-seeking within our environment. The information we sample through this process is then used to update the relevant schema, so our future understanding of situations will be strongly influenced by what we have encountered in the past. The perceptual cycle model and schema theory have been applied to various topics within human factors and ergonomics (Plant and Stanton, 2013a, 2013b; Stanton et al., 2009), including RLX safety (Salmon et al., 2013b). It has been argued that inappropriate decisions and actions at RLXs can be explained as schema-driven failures; for instance, if a driver has encountered a specific RLX multiple times without seeing a train, they may form a strong expectation that they will never see a train there. This type of faulty schemata can have tragic consequences, as in the 2007 collision near Kerang, Victoria, Australia, in which a truck driver failed to detect that flashing lights were activated and a train was approaching. In the Kerang collision, the truck driver had extensive experience with the RLX in an inactive state, which contributed to him making a "looked-but-failed-to-see" error and not realising the RLX signals were active (Salmon et al., 2013b). Inappropriate schema activation can also occur (Norman, 1981). Here the RLX environment may inadvertently trigger the wrong schemata in 
drivers; for example, drivers may activate the schemata for a standard active RLX if they do not possess relevant schema for stop-controlled RLXs, which in turn may lead to the driver failing to stop and/or adequately scan for trains.

At stop-controlled RLXs, different drivers may possess vastly different schemata that guide their actions. Drivers who have extensive experience at passive RLXs may possess schema that indicate stop signs are unnecessary (e.g., as in the US example, where stop signs are used indiscriminately, or in Australia if drivers do not appreciate the potential risks), which could lead them to violate the warnings. Alternatively, drivers in Australia may possess schemata that a stop sign signals a specific subtype of RLX (i.e., no active warnings, restricted visibility), which should prompt more cautious behaviour. On the other hand, drivers who are relatively unfamiliar with stop-controlled RLXs, or passive RLXs more broadly, may not expect to encounter a stop sign in that environment, and may instead be searching for flashing lights to guide their decision-making (Salmon et al., 2013a). These drivers may experience difficulty or confusion when negotiating the RLX.

\subsection{Crash risk at stop-controlled RLXs}

Most published comparisons of crash risk between crossbuck-only and stop-controlled RLXs have utilised US data. Some analyses conclude that, compared with crossbuck-only or yield signs, stop controls are associated with reduced injury risk (Eluru et al., 2012) and lower crash rates (Millegan et al., 2009; Saccomanno et al., 2007; Yan et al., 2010a, 2010b). Other studies find that crash rates are equivalent (McCollister and Pflaum, 2007) or even increased at stop crossings (Austin and Carson, 2002; Raub, 2009). The reason for this inconsistency is unclear, but may be due to non-compliance (e.g., if stop signs are installed at more dangerous RLXs, but drivers ignore them, crash rates will be higher).

\subsection{The current study}

The current study aimed to understand drivers' reasons for non-compliance at passive stopcontrolled crossings, as first step to identifying possible avenues for improving safety. Participants drove an instrumented vehicle around a pre-determined route that incorporated three stop-controlled RLXs and seven other RLXs. Multiple objective and subjective data sources (e.g., vehicle parameters, visual checking behaviour, video footage, concurrent think aloud protocols, post-drive interviews) were combined to provide a comprehensive understanding of the reasons for drivers' compliance or noncompliance at stop-controlled RLXs. 


\section{Method}

\subsection{Participants}

Twenty-two drivers (11 novice, 11 experienced) participated voluntarily and were financially reimbursed for their time. Novice drivers (19-21 years) had 12-24 months driving experience. Experienced drivers (33-55 years) had at least 10 years driving experience. Table 2 shows the sample demographic characteristics. Participants were recruited through local newspapers, noticeboards, community groups and word-of-mouth. All lived and/or worked locally and regularly drove in the region. Ethical aspects of the research were approved by Monash University Human Research Ethics Committee (CF12/0077-2012000016).

Table 2

Demographic characteristics of participants.

\begin{tabular}{lccc}
\hline & Novice & Experienced & Difference \\
\hline $\begin{array}{l}\text { Sex } \\
\text { Age (years) }\end{array}$ & 6 females, 5 males & 6 females, 5 males & n/a \\
& $19.3(0.6)$ & $45.1(7.6)$ & $t(10.1)=-11.23, p<.001$, \\
& & $R^{2}=.93$ \\
$\begin{array}{l}\text { Driving experience } \\
\text { (years) }\end{array}$ & $1.6(0.3)$ & $27.3(7.6)$ & $t(10.0)=-11.28, p<.001$, \\
$\begin{array}{l}\text { Driving exposure } \\
\text { (hours per week) }\end{array}$ & $6.6(7.9)$ & $17.1(14.5)$ & $t(15.5)=-2.10, p=.052$, \\
\end{tabular}

Note. $t$-tests computed with equal variances not assumed because Levene's Test for Equality of Variances was significant for all comparisons.

\subsection{Route and target $R L X$ types}

The study route was $30.5 \mathrm{~km}$ around Bendigo, a regional city approximately $150 \mathrm{~km}$ north-west of Melbourne, Australia. The region encompasses three commuter and freight train lines and contains a high density of RLXs. The route incorporated urban and rural roads with varying environments, terrain and speed limits. Drivers crossed three stop-controlled RLXs (see Figure 2) and seven additional RLXs (one passive, six active). The focus here is on the stop-controlled RLXs only, as in previous work we have analysed data from the other RLX types (Lenné et al., 2013a, 2013b; Salmon et al., 2013a, 2014a).

2.2.1. Stop-controlled $R L X 1$. This $R L X$ was encountered $10 \mathrm{~km}$ into the route, travelling northwest on an unsealed gravel road in a rural-residential area. The speed limit was $50 \mathrm{~km} / \mathrm{h}$, although there were no visible speed signs on approach. The road curves for $1 \mathrm{~km}$ but is straight for $100 \mathrm{~m}$ approaching the RLX, then ends in a T-intersection $40 \mathrm{~m}$ after the RLX. At the RLX there were four signs: a rectangular 
sign with a white crossbuck on a red background; a stop sign; a "LOOK FOR TRAINs" sign; and a pair of "RAILWAY CROSSING" signs flanking the road. On approach, there was an advisory sign indicating the stop sign ahead. There were no road markings related to the RLX. Visibility along the train line was obscured by foliage on both sides.

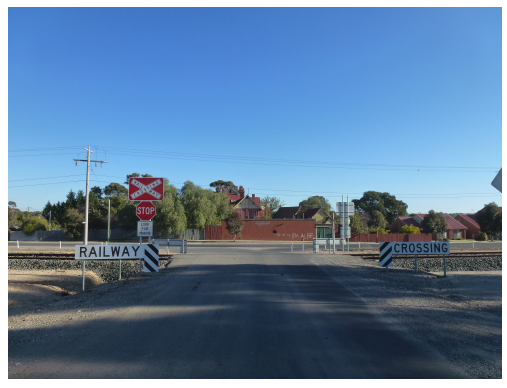

RLX 1

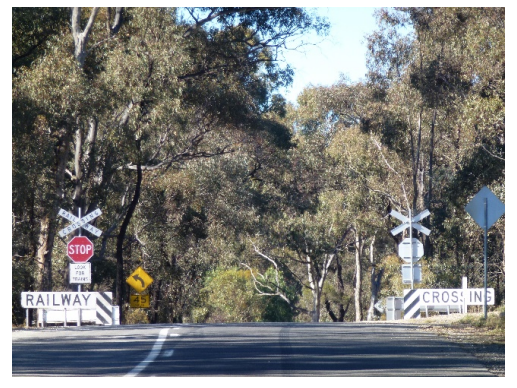

RLX 2

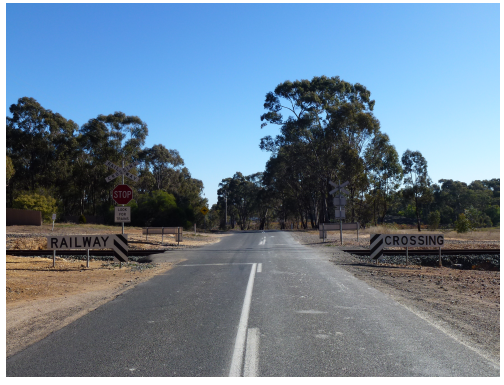

RLX 3

Figure 2. Approach views of the three stop-controlled rail level crossings (RLXs) in the on-road test route.

2.2.2. Stop-controlled $R L X 2$. This RLX was encountered $23.6 \mathrm{~km}$ into the route, travelling southeast on a sealed road in a rural area. The speed limit on the road was $80 \mathrm{~km} / \mathrm{h}$ but dropped to $60 \mathrm{~km} / \mathrm{h}$ shortly before the RLX. The road is mainly straight with some gentle curves for $1.5 \mathrm{~km}$ and is completely straight for $130 \mathrm{~m}$ approaching the RLX. At the RLX there were four signs: a white crossbuck; a stop sign; a "LOOK FOR TRAINS" sign; and a pair of "RAILWAY CROSSING" signs flanking the road. On approach, there was an advisory sign indicating the stop sign ahead, and faded road markings that said "RAIL X". Road markings at the RLX were a painted white stop line and double white centre dividing lines. Visibility along the train line was obscured by foliage on both sides on approach, and by an embankment on the right.

2.2.3. Stop-controlled $R L X$ 3. This RLX was encountered $26 \mathrm{~km}$ into the route, travelling northwest on a sealed road. (This was the same crossing as RLX 2, traversed in the opposite direction.) The speed limit was $60 \mathrm{~km} / \mathrm{h}$ on approach to the RLX. Participants turned onto the road $350 \mathrm{~m}$ before the RLX, which is straight for 170 m approaching the RLX. At the RLX there were four signs: a white crossbuck; a stop sign; a "LOOK FOR TRAINS" sign; and a pair of "RAILWAY CROSSING" signs flanking the road. On approach, there was an advisory sign with an image of a steam train. Road markings at the RLX were a painted white stop line and double white centre dividing lines; there were no visible RLX road markings on approach. Visibility along the train line was obscured by foliage on both sides. 


\subsection{Instrumented Vehicle}

Participants drove Monash University Accident Research Centre's On-Road Test Vehicle, an instrumented vehicle that collects vehicle-related data and records in-vehicle and external events using seven cameras. Eye and head movements were measured using an Arrington Binocular Scene Camera $60 \mathrm{~Hz}$ head-mounted system. Each driver's gaze was calibrated using a nine-point calibration grid prior to the drive. Due to ambient light variations, calibration quality was insufficient to code eye movements, so only gross head movements were analysed.

\subsection{Procedure}

Participants were not informed that the research focused on RLXs, but rather were told that the aim of the study was to examine how drivers respond to different road environments (e.g., town centre vs. rural highway vs. gravel road). Participants provided written informed consent, completed a brief demographic questionnaire, and received 10-15 minutes' training in the concurrent verbal protocol technique. This began in the interview room, where a researcher explained the "think aloud" task with extensive examples of the types of verbalisations that they should make. The participant was then taken to the instrumented vehicle and asked to familiarise themselves with the vehicle and set up the driver's seat, mirrors and eye-tracker. Participants completed a 10-minute practice drive, with further concurrent verbal protocol training: for the first 2 minutes a researcher provided an example concurrent verbal protocol, and for the remainder of the practice drive the participant provided the concurrent verbal protocol, with the researcher providing feedback as appropriate.

The test route took around 40 minutes and began once the participant was comfortable driving the vehicle. Two researchers accompanied the participant: one in the front seat providing navigation instructions, and one in the back seat running the data collection systems. Throughout the test route participants provided a concurrent verbal protocol to support assessment of their situation awareness (Salmon et al., 2014b) and were prompted to resume their verbal protocol if they became silent for extended periods. A recent on-road study undertaken to test the impact of providing a concurrent verbal protocol on driver behaviour found no adverse impacts on driving performance (Salmon et al., 2017).

After the drive participants completed two Critical Decision Method (CDM) interviews (Klein et al., 1989). Each CDM focused on a single RLX encounter, which yielded a total of 12 interviews about stop-controlled RLXs (and 32 focusing on other RLXs). CDM has been used to examine naturalistic decision making across a range of domains (Hoffman et al., 1998; Plant and Stanton, 2013; Stanton et al., 2007), including RLXs (Mulvihill et al., 2016). Although CDM was developed to study expert decisionmaking, it has been successfully adapted to examine decision-making while driving (Beanland et al., 
2016; Stanton et al., 2007; Walker et al., 2009; Young et al., 2015). At the beginning of each interview, the participant was asked to describe a specific RLX encounter in as much detail as possible. They were then asked to summarise the situation, including the decision they made. Following this, the interviewer used a series of prompts designed to assist recall of past events and explore factors that influenced the individual's decision (e.g., personal goals, environmental cues, information use, experience) and their situation awareness (for a full list of prompts used, see Read et al., 2017). The interviewer took notes during the drive to further prompt recall and clarify comments as required (e.g., "during the drive you said you didn't think this train line was used.")

\subsection{Data Analysis}

Data were recorded continuously throughout the route, but for the current analyses $110 \mathrm{~m}$ segments were extracted, encompassing $100 \mathrm{~m}$ on approach and $10 \mathrm{~m}$ after each RLX. The approach distance of $100 \mathrm{~m}$ was selected as this comprised a straight section of road for all crossings. At this point drivers could see the RLX ahead, but did not have adequate sight distance to detect an oncoming train with enough time to stop. Three data sources were analysed for each segment: travel speed $(\mathrm{km} / \mathrm{h})$; visual checks; and verbal protocols. Visual checks were defined as a head movement $>30^{\circ}$ from straight ahead, during which the participant was looking outside the vehicle, on approach to the RLX or at the stop line.

Participants' verbal protocols and CDMs were transcribed verbatim and then coded by two independent raters. For verbal protocols, each RLX segment was coded for key concepts, which were derived by initially reviewing transcripts and noting elements that drivers mentioned, then grouping these in themes. Only concepts directly relevant to the RLX were coded. This resulted in 10 concepts under two broad themes: the decision made (stopping, slowing, not stopping) and factors that influenced drivers' decisions (rail level crossing, stop sign, visual information, auditory information, absence of trains, absence of active controls, and safety). CDM transcripts were coded for concepts relating to the driver's goals, use of available information, key influencing factors and experience that informed the decision, and alternative courses of action, to understand how they made the decision of whether to stop or proceed and how they rejected alternative actions. Inter-rater agreement was $94 \%$ $(\mathrm{k}=.92)$ for verbal protocols and $92 \%(\mathrm{k}=.91)$ for CDMs.

\section{Results}

\subsection{Traffic conditions}

Across 22 participants there were 66 encounters at stop-controlled RLXs. Inspection of forwardfacing camera footage from the instrumented vehicle revealed no situations in which participants' 
driving behaviour was directly influenced by other road users (e.g., lead vehicle stopping, requiring the participant to stop), and few situations with potential for indirect influence (e.g., another vehicle proceeding through at speed, prompting the participant to behave similarly). No trains were present during any crossings, and during 54 crossings (82\%) participants did not encounter any other traffic. When traffic was present it was minimal, typically a vehicle travelling in the opposite direction $(n=8)$ or cyclists on the roadside $(n=2)$. Most of these other road users did not cross the RLX, or crossed after the participant. There was only one case in which another driver crossed in the opposite direction immediately before a participant; this other driver failed to stop but the participant came to a complete stop.

\subsection{Approach speeds and stopping behaviour}

Participants came to a complete stop $(0 \mathrm{~km} / \mathrm{h})$ at $59 \%$ of crossings. On a further $27 \%$ of crossings drivers executed a rolling stop, defined as a minimum speed above $0 \mathrm{~km} / \mathrm{h}$ but below $10 \mathrm{~km} / \mathrm{h}$. On the remaining 9 crossings (14\%) drivers violated the stop controls, with minimum travel speeds up to $56.4 \mathrm{~km} / \mathrm{h}$. Of these 9 incidents, 4 involved experienced drivers and 5 involved novice drivers. There was a trend whereby experienced drivers were more likely to stop completely, and novice drivers were more likely to make rolling stops, but the association between driving experience and stopping behaviour was not statistically significant, $\chi^{2}(2)=3.37, p=.186$. As travel speeds (and visual checks, see section 3.3) did not differ between novice and experienced drivers, driving experience was not included as a variable in subsequent statistical analyses.

Most drivers behaved consistently across RLXs: 41\% were compliant ( 9 drivers: 7 experienced, 2 novice), i.e., they always stopped completely; 36\% were cautious ( 8 drivers: 2 experienced, 6 novice), i.e., they always made at least a rolling stop; and 9\% were consistently non-compliant (2 drivers: 1 experienced, 1 novice), i.e., they violated at all three RLXs. Only 14\% were inconsistently non-compliant (3 drivers: 1 experienced, 2 novice), i.e., they were non-compliant at only one RLX.

Figure 3 illustrates the relationship between approach speeds 100m from the RLX and subsequent stopping behaviour. Among the full sample there was no significant correlation between approach speeds and stopping behaviour, $r(66)=.11, p=.367$. However, when including only violators, there was a significant large correlation between approach speeds $100 \mathrm{~m}$ away and minimum speed through the $\operatorname{RLX}, r(9)=.87, p=.002$. This suggests that approach speeds do not predict the intention to violate, but among drivers who do violate, their approach speed reflects the speed at which they negotiate the intersection. 


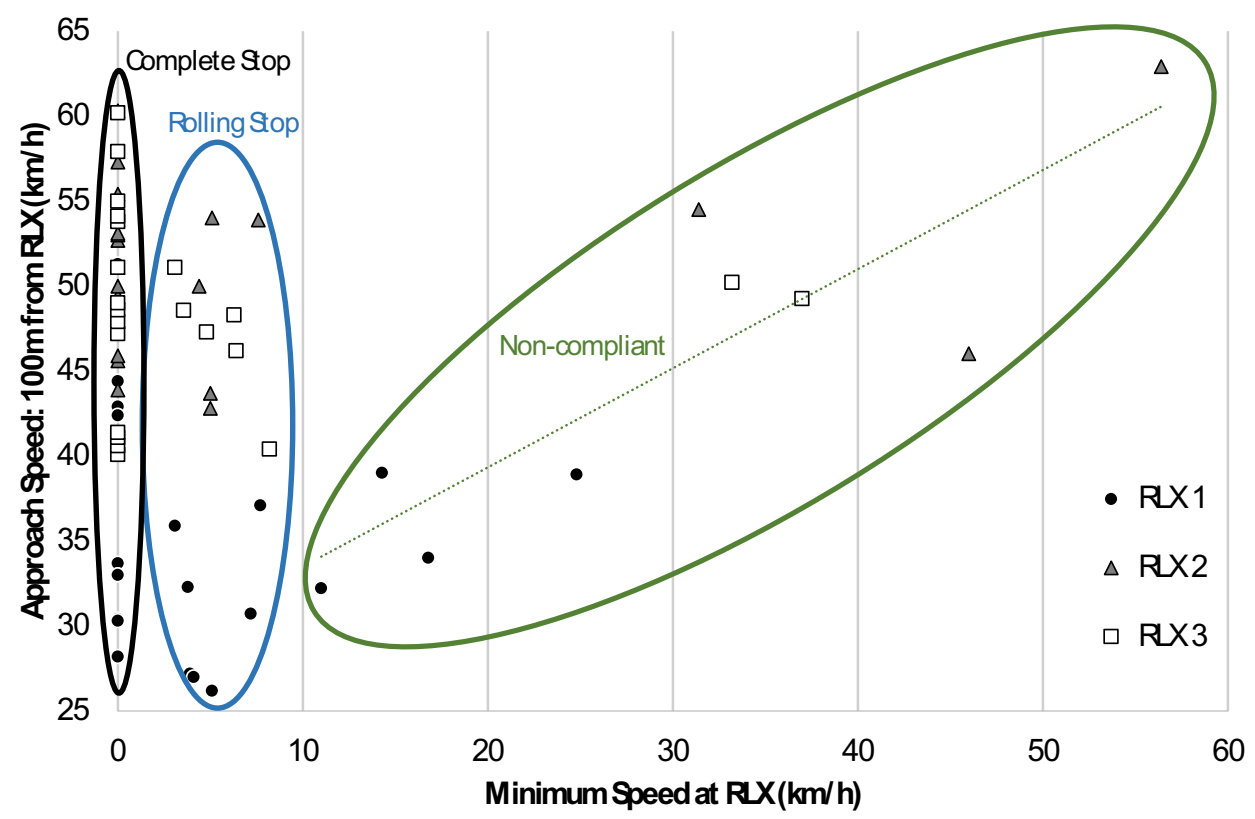

Figure 3. Relationship between approach speed (i.e., travel speed 100m from the RLX) and stopping behaviour at three stop-controlled RLXs. Ovals indicate the three types of stopping behaviour used in statistical analyses: "complete stop" indicates a minimum speed of $0 \mathrm{~km} / \mathrm{h}$; "rolling stop" indicates that the driver decelerated to a minimum speed of $<10 \mathrm{~km} / \mathrm{h}$ before accelerating and proceeding through the crossing; and "non-compliant" indicates that the driver proceeded through the RLX with a minimum speed above $10 \mathrm{~km} / \mathrm{h}$. Dotted green line represents the linear trend line for the association between approach speed and minimum speeds for non-compliant drivers.

Figure 4 shows speed profiles throughout each 110m RLX segment. Each RLX was analysed separately as approach speeds differed between crossings. Average speeds were binned into $10 \mathrm{~m}$ segments and then compared between drivers who violated the stop controls (i.e., minimum travel speed $>10 \mathrm{~km} / \mathrm{h}$ ) and those who made complete or rolling stops (the latter groups were combined as their speed profiles were similar). Each $10 \mathrm{~m}$ segment was compared using $t$-tests, to reveal the first point at which violators and compliant drivers exhibited significantly different speeds. 

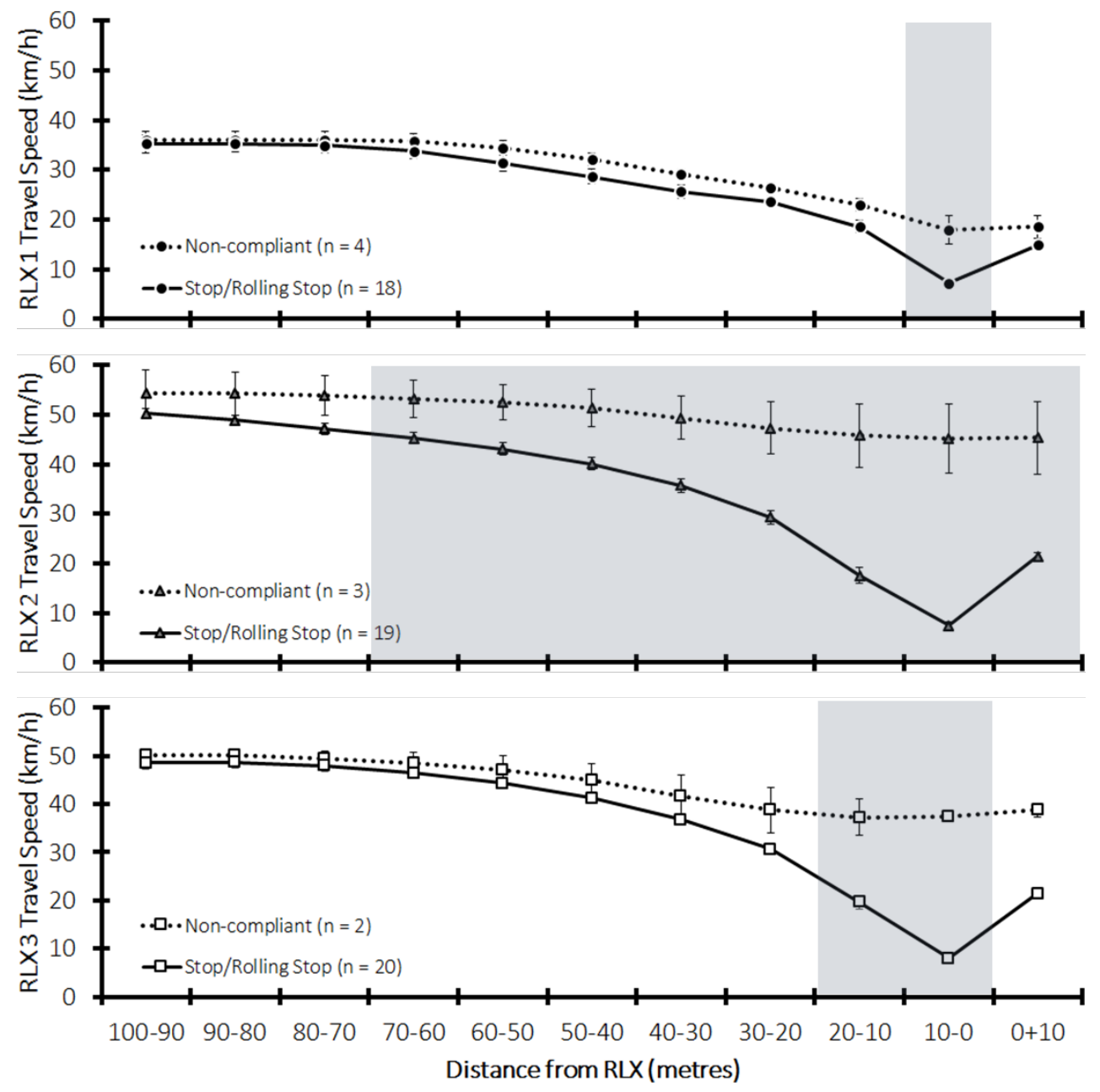

Figure 4. Speed profiles for compliant (stop/rolling stop) and non-compliant drivers when negotiating three stop-controlled RLXs. Shaded segments indicate points when travel speeds were significantly higher for non-compliant drivers. Error bars represent \pm 1 standard error of the mean.

For RLX 1, speeds were only significantly different in the period 10-0m before the RLX, $t(3.2)=3.66, p=.032, M_{\text {diff }}=10.8 \mathrm{~km} / \mathrm{h}, S E=2.9,95 \% \mathrm{Cl}[1.6,19.9]$. For RLX 3, compliant drivers first exhibited significantly lower speeds during the period $20-10 \mathrm{~m}$ before the $\operatorname{RLX}, t(20)=3.57, p=.002$, $M_{\text {diff }}=17.6 \mathrm{~km} / \mathrm{h}, S E=4.9,95 \% \mathrm{Cl}[7.3,27.9]$, and then continued to maintain lower speeds until $10 \mathrm{~m}$ after the $\mathrm{RLX}, t(20)=6.28, p<.001, M_{\text {diff }}=17.3 \mathrm{~km} / \mathrm{h}, \mathrm{SE}=2.8,95 \% \mathrm{CI}[11.5,23.0]$. In contrast, differences in travel speeds began much earlier at RLX 2, with the first significant difference during the period 70 $60 \mathrm{~m}$ before the $\mathrm{RLX}, t(20)=2.06, p=.025, M_{\text {diff }}=6.8 \mathrm{~km} / \mathrm{h}, \mathrm{SE}=2.8,95 \% \mathrm{CI}[1.1,15.0]$. These discrepant patterns are likely the result of the "pre-approach" road environment: RLX 1 was at the end of a gravel road and RLX 3 occurred 350m after an intersection, so travel speeds were relatively low even before the final $100 \mathrm{~m}$ approach to the RLX, whereas RLX 2 was on a longer road where the speed limit dropped from $80 \mathrm{~km} / \mathrm{h}$ to $60 \mathrm{~km} / \mathrm{h}$ within the last $500 \mathrm{~m}$. 


\subsection{Visual checking}

The number, duration and timing of visual checks made on approach to each RLX was analysed using generalised estimating equations, an extension of the general linear model that permits analysis of repeated measurements with varying conditions. This approach was adopted as some drivers exhibited different stopping behaviour at each RLX. The analysis used a linear model with and exchangeable correlation matrix, with stopping behaviour (complete stop, rolling stop, non-compliant) and RLX (1, 2, 3) as factors. Reference groups were "complete stop" for stopping behaviour and "RLX 1 " for RLX.

The number of visual checks made ranged from 0 to 10 , and varied significantly according to both $\mathrm{RLX}, \chi^{2}(2)=15.43, p<.001$, and stopping behaviour, $\chi^{2}(2)=19.85, p<.001$, but the interaction between factors was not significant (see Figure 5). Non-compliant drivers made significantly fewer visual checks than those who stopped completely $\left(B=-2.26, S E=0.66, \chi^{2}=11.68, p=.001\right)$, and the sole driver who made no visual checks was non-compliant. However, there was no difference in number of visual checks made when comparing drivers who made complete stops vs. rolling stops $(B=0.05$, $\left.S E=0.38, \chi^{2}=0.02, p=.887\right)$. Drivers executed fewer visual checks at RLX 1 compared to both RLX 2 $\left(B=1.18, S E=0.38, \chi^{2}=9.96, p=.002\right)$ and $\operatorname{RLX} 3\left(B=0.98, S E=0.38, \chi^{2}=9.03, p=.003\right)$.

The total duration of visual checks made ranged from 1.6 to 16.7 seconds, and varied significantly with $\operatorname{RLX}, \chi^{2}(2)=17.35, p<.001$, and stopping behaviour, $\chi^{2}(2)=18.27, p<.001$ (see Figure 5 ). As with number of visual checks, non-compliant drivers spent significantly less time making visual checks than those who stopped completely $\left(B=-2.45, S E=0.72, \chi^{2}=11.76, p=.001\right)$, but there was no difference between drivers who made complete stops vs. rolling stops $\left(B=-0.39, S E=0.74, \chi^{2}=0.28\right.$, $p=.597)$. Duration of visual checks was also shorter at $\operatorname{RLX} 1$ compared with both $\operatorname{RLX} 2(B=1.89$, $\left.S E=0.59, \chi^{2}=10.18, p=.001\right)$ and $\operatorname{RLX} 3\left(B=1.69, S E=0.48, \chi^{2}=12.20, p<.001\right)$. 


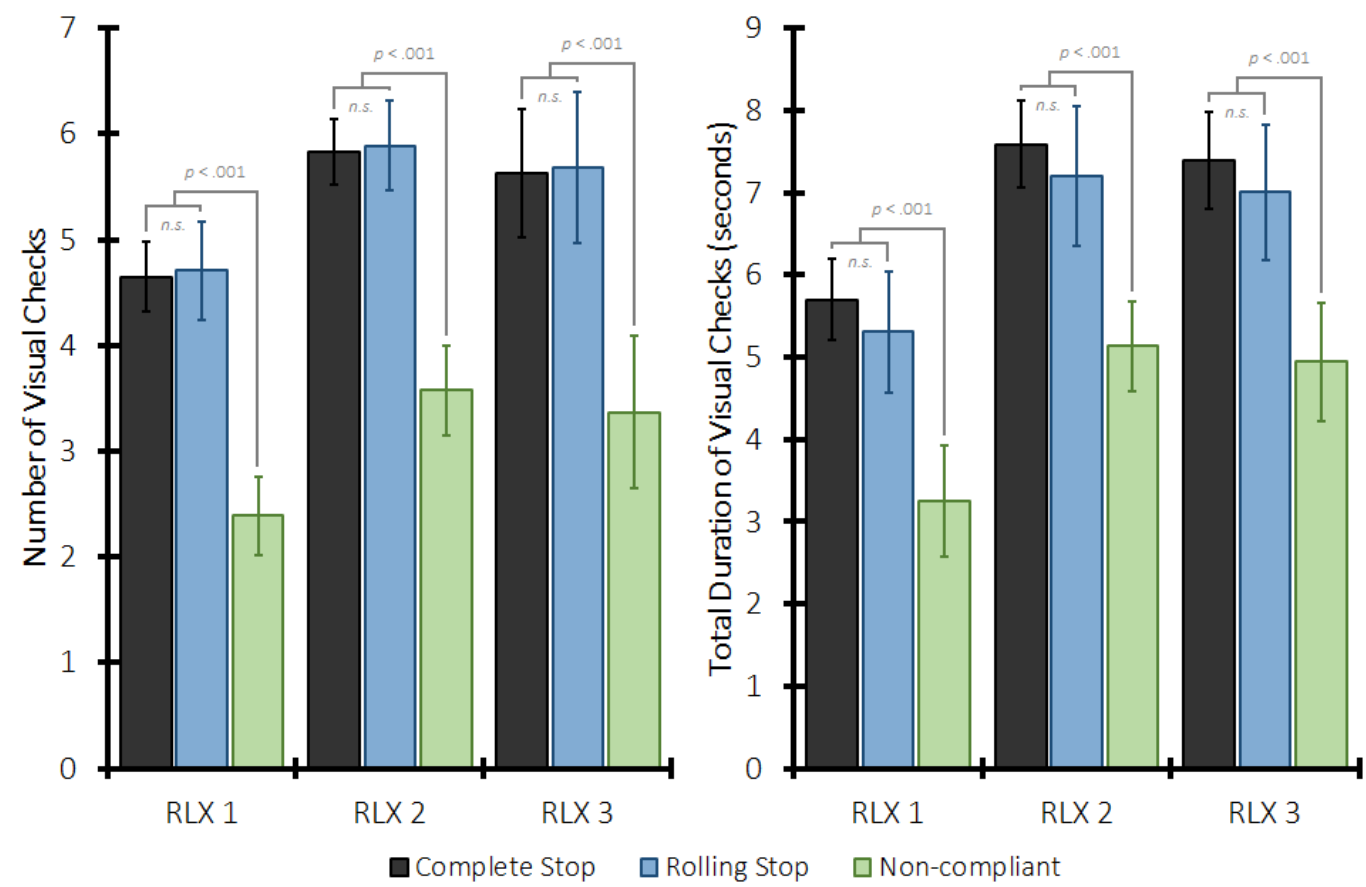

Figure 5. Average number (left panel) and duration (right panel) of visual checks made by drivers at three stop-controlled level crossings. Error bars represent \pm 1 standard error of the mean. Noncompliant drivers (i.e., those proceeded through with a minimum speed $>10 \mathrm{~km} / \mathrm{h}$ ) made fewer visual checks and spent less time checking for trains. Visual checking behaviour was not significantly different between drivers who made complete vs. rolling stops.

The timing of the first and last visual checks made was analysed as time in seconds relative to the point at which drivers first reached the stop line before the RLX. (As RLX 1 did not have a stop line, this was measured as the equivalent distance from the tracks.) As such, positive values indicate visual checks made before reaching the stop line, and negative values indicate visual checks made after reaching the stop line (see Figure 6). Participants made their first visual check an average of 4.6 seconds $(S D=4.0)$ before the stop line, and this timing did not vary between RLXs, $\chi^{2}(2)=0.16, p=.924$, or with stopping behaviour, $\chi^{2}(2)=2.28, p=.320$, although there was a trend towards non-compliant drivers beginning their visual checks sooner.

Participants made their final visual check an average of 3.1 seconds $(S D=1.9)$ after reaching the stop line; as most drivers stopped completely, this occurred while they were still stopped or while they were in the process of crossing the tracks. The timing of the final visual check varied with stopping behaviour, $\chi^{2}(2)=33.51, p<.001$ (see Figure 6). Non-compliant drivers made their final visual check significantly earlier than those who stopped completely $\left(B=3.15, S E=0.55, \chi^{2}=33.31, p<.001\right)$. Drivers who made rolling stops also completed their visual checks earlier than those who stopped completely $\left(B=0.79, S E=0.36, \chi^{2}=4.72, p=.030\right)$. Timing of the final visual check did not differ between RLXs, $\chi^{2}(2)=0.81, p=.668$. 


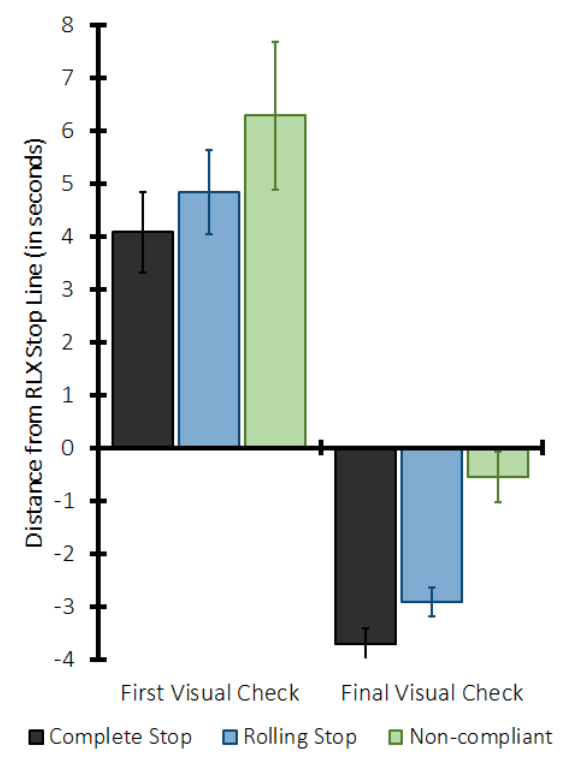

Figure 6. Timing of first and final visual checks on approach to RLXs, measured relative to the time at which the driver first reached the stop line. Error bars represent \pm 1 standard error of the mean.

\subsection{Verbal protocol analysis}

Participants' concurrent verbal protocols on approach to stop-controlled RLXs were coded for key concepts; the results are shown in Figure 7. When drivers executed a complete stop the most common concepts mentioned were: the RLX itself (e.g., "there's a railway crossing coming up"); visual information (e.g., "look both ways, there's no trains coming", "my vision to the right isn't very good"); the stop sign (e.g., "there's a stop sign on the railway crossing"); stopping (e.g., "I'll slow right down, I'm not that familiar with the crossing. I'll stop, actually"); and the absence of trains (e.g., "doesn't seem like there's any trains"). Explicit mentions of safety (e.g., "it's all safe") were rare and were only made by drivers who executed a complete stop. When drivers executed rolling stops, the most common concepts mentioned were again the RLX, visual information, the absence of trains, stopping, and the stop sign.

Non-compliant drivers mentioned fewer concepts on average than drivers who made rolling or complete stops, as shown in Figure 7. The most frequently mentioned concepts by non-compliant drivers were visual information (56\%) and the RLX itself (56\%). Less than half the violators (44\%) acknowledged the stop sign, and only two (22\%) mentioned the absence of a train. Two violators also explicitly mentioned that they did not want/intend to stop before they proceeded through the RLX (e.g., "they want me to stop for but to tell you the truth I don't think I'm going to."). 


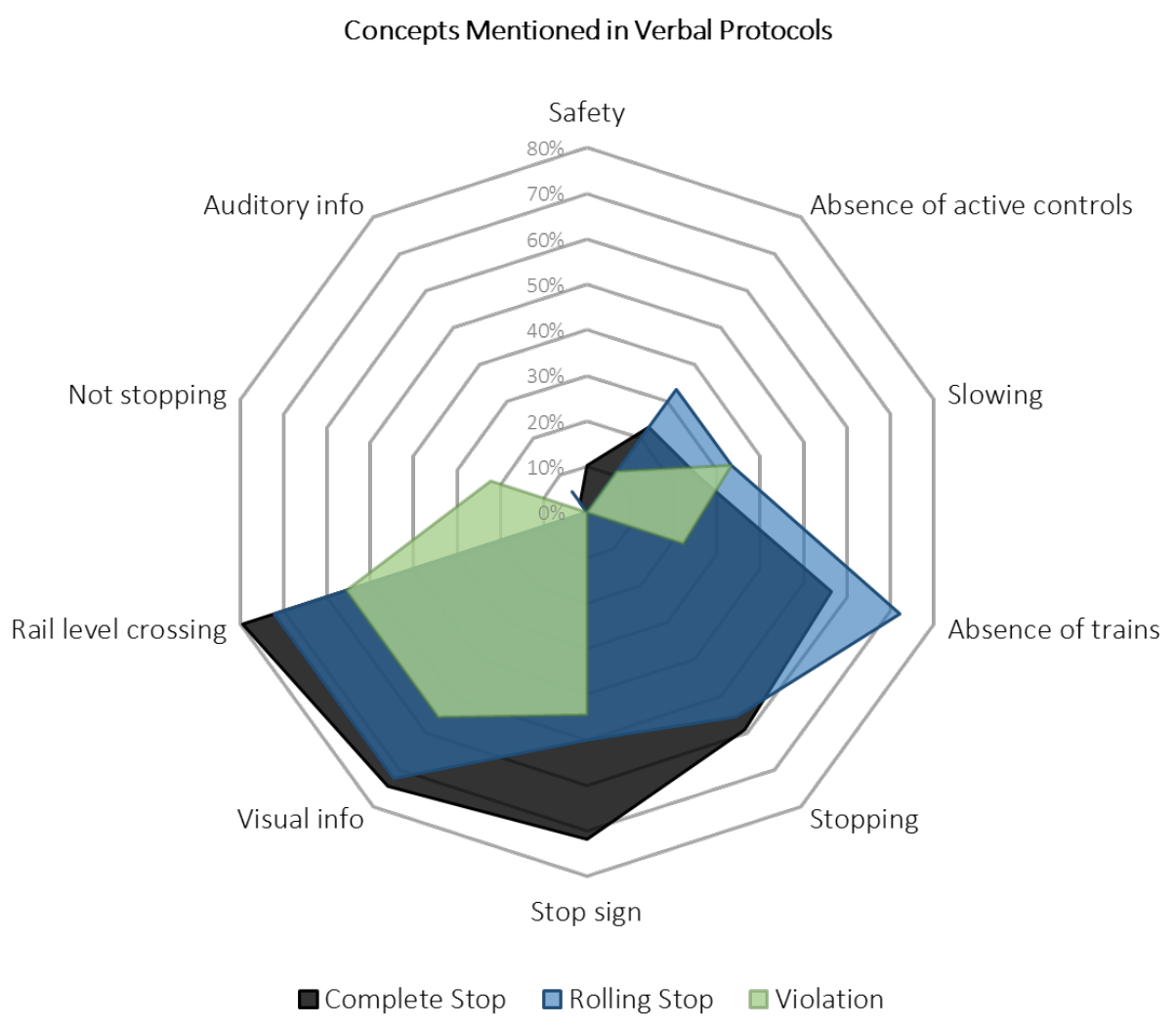

Figure 7. Radar plot depicting the percentage of drivers who mentioned specific concepts on approach to stop-controlled RLXs, according to stopping behaviour: Complete Stop $(n=39)$; Rolling Stop $(n=18)$; or Violation ( $n=9$ ).

\subsection{Post-drive CDM interviews}

Twelve drivers completed post-drive CDM interviews regarding one of their encounters at a stop-controlled RLX. This included: 5 complete stops (4 experienced, 1 novice); 2 rolling stops (both novice); and 5 non-compliant encounters (2 experienced, 3 novice).

3.5.1. Complete stops. The five CDMs with drivers who stopped completely all focused on RLX 1 . These drivers' primary goals were to cross the RLX safely and/or to stop and check for trains. The most important influencing factor, mentioned by all drivers who stopped completely, was the stop sign itself. All drivers opined that they had used all available information to make their decision, and most experienced drivers reported that no other information was required, with one suggesting an advance warning sign and/or other vehicles (e.g., stopped on the other side of the RLX) would be helpful. The novice driver indicated that flashing lights (but not necessarily a boom gate) would be a useful addition.

Experienced drivers reported drawing on general driving experience to assist their decisionmaking, including experience at other stop-controlled intersections (i.e., road intersections) and general 
knowledge of road rules, even though they had experience with passive RLXs. In contrast, the novice driver reported drawing on experience with other passive RLXs. Most drivers said stopping was an automatic decision with no time pressure; "it was a no brainer". Only one driver felt time pressure, because the car gathered speed downhill and was going faster than they were comfortable with.

Drivers who stopped completely suggested that stopping at the RLX was the only viable option, although it is theoretically possible to ignore the stop sign. One driver indicated they might consider proceeding through without stopping if in a hurry, whereas others suggested they would only proceed without stopping if there was no stop sign, or if it was an emergency (e.g., a vehicle close behind was about to hit them). Drivers mentioned the need to obey road rules (e.g., "You have to do what the law says."), with some linking this to enforcement (e.g., "I've only got one demerit point left. I need to do everything right.") Some experienced drivers understood the stop sign was installed for a reason: "you obviously see the stop sign and realise that there's a reason for it, and that would be that it's difficult to see". Drivers suggested that even without the stop sign they would slow down considerably, and possibly stop completely, because of the RLX.

3.5.2. Rolling stops. The two rolling stop CDM interviews were with novice drivers who proceeded through RLX 1 with minimum speeds of $3.9 \mathrm{~km} / \mathrm{h}$ and $7.2 \mathrm{~km} / \mathrm{h}$. Both drivers believed they had stopped completely and their responses were similar to those drivers who did stop completely. Specifically, their stated goals were to cross safely and to stop at the RLX and check for trains; the stop sign was the most influential cue in their decision-making; and they considered it an automatic decision with no time pressure. Both drivers believed they used all the information available, but said additional information would have been helpful. One driver suggested active controls (e.g., "Lights...and having boom gates. So you'd know from a distance how far away the train is") and the other suggested an advance warning sign that explicitly mentioned that the stop sign was at an RLX (e.g., "I was confused whether [the stop sign] was before or after the tracks. Yes, stop at the railway crossing or something, yes, to make it a bit more clear").

When describing how they drew on previous experience, one mentioned general driving experience, the other mentioned knowledge of road rules, and both mentioned experience with stop signs in general: "Just having to stop at the stop signs. So you know when you see one, you have to stop no matter what." They were more forthcoming with alternative actions than drivers who stopped completely, with both suggesting that they could have proceeded through without stopping, but they chose to stop because they are law abiding. One driver suggested they could have re-routed to avoid the RLX altogether.

3.5.3. Unintentional non-compliance. The five CDM interviews with non-compliant drivers included three who reported unintentional non-compliance and two intentional violations. These drivers 
differed in situation awareness and decision-making, consistent with Reason et al.'s (1990) distinction between errors and violations having unique psychological underpinnings, and so are described separately.

Three drivers described unintentional non-compliance at RLX 1, with minimum speeds of 11.0, 14.3 and $16.8 \mathrm{~km} / \mathrm{h}$. All failed to notice the stop sign, and suggested this was because they were focused on other aspects of the environment. These included the RLX itself, looking for trains, the road surface, the T-intersection after the RLX and other road users at or near the RLX. Their stated goals were to look for trains and cross safely. The primary factors influencing their decisions varied between individuals, but included the RLX itself (e.g., tracks, "LOOK FOR TRAINs" sign), the absence of a train, safety, and the presence of other road users. All indicated they habitually slow for RLXs.

None of the drivers reported experiencing time pressure, and indicated it was a relatively quick or automatic decision to slow and then proceed. One experienced driver opined that she used all information that she attended to and did not require any other information, as she relies on her own assessment of the situation rather than RLX signs and signals. The other two drivers said that they used all information except the stop sign, and believed that active controls would be a useful addition (the experienced driver suggested flashing lights, while the novice drivers suggested both lights and booms). One driver added that clearing vegetation would help by improving visibility, and another suggested improving the road surface.

To help make their decision, drivers reported drawing on their experience of learning to drive (e.g., the novice driver stated that their mother always made them stop at passive RLXs) and general driving experience. The novice driver mentioned drawing on previous experience with other passive RLXs, whereas the experienced drivers did not mention drawing on any knowledge or experience that was specifically related to RLXs.

When asked to describe possible alternative actions, all drivers suggested that stopping completely would be an option but the two experienced drivers affirmed they had made the appropriate decision: stopping was unnecessary ("it didn't warrant a full stop"), but a faster travel speed would be unsafe. Neither of these drivers executed full stops at the other stop-controlled RLXs; one driver made rolling stops at RLX 2 and 3, whereas the other driver committed violations. The novice driver indicated they would stop if there was a train and might adopt a faster travel speed if the road surface was better and they knew there was no train coming (i.e., based on familiarity with train schedules).

3.5.4. Intentional violations. The two drivers who committed intentional violations negotiated RLX 2 with minimum speeds of 46.0 and $56.4 \mathrm{~km} / \mathrm{h}$. Both acknowledged the stop sign during their verbal 
protocols. They reported slowing slightly and looking and listening for trains. Their stated goals were to cross safely without slowing too much: "it would require a lot of time beforehand to slow all the way down to a complete stop and speed all the way back up again. So you may as well keep going."

Seeing the stop sign and the RLX provided a cue that they needed to decide whether to stop, and after visually scanning the environment both drivers concluded stopping was unnecessary. Both nominated the absence of trains as the primary factor influencing their decision. They described visibility at the RLX as good and stated they had adequate time to ensure that no trains were approaching. Both reported that they used all available information, but one suggested the addition of flashing lights would be helpful.

One of the drivers reported experiencing "a bit" of time pressure in making their decision and was surprised by the speed at which they drove through the RLX; this driver stopped completely at RLX 1 and made a rolling stop at RLX 3 . The other driver reported no time pressure and felt the decision was appropriate; this driver also committed violations at RLX 1 and 3.

When asked about previous experience that informed their decision, both drivers mentioned having extensive experience driving across RLXs, including passive RLXs. Both noted that in the local area RLXs are common but trains are rare, which affected their decision-making: "I don't have much experience of trains. I've never actually stopped at a crossing... [In Bendigo] you cross railway tracks relatively frequently but you rarely, rarely have to stop."

When asked to consider alternative possible actions, both drivers suggested that they could have stopped. One also suggested several other alternatives, including re-routing or turning off the road if a train was approaching.

\section{Discussion}

The current study provided an in-depth analysis of driver behaviour at stop-controlled RLXs. The decisions made by participants can be broadly categorised as complete stops, rolling stops, unintentional non-compliance, and intentional violations. This variability reinforces the notion that even when faced with the same RLX infrastructure, drivers may interpret the situation differently and exhibit vastly discrepant behaviours. The key findings from each form of decision are discussed below.

\subsection{Compliant and cautious drivers}

The most common behaviours observed in the current study were complete (59\%) and rolling stops (27\%), which participants indicated was an automatic rule-based decision. Interestingly, some of the drivers who made rolling stops believed that they had stopped completely. Consistent with this, 
there were few differences between drivers who made complete and rolling stops, especially with respect to travel speeds and duration of visual checks. Participants who stopped completely appeared more focused on the stop sign and emphasised that stopping completely was the only sensible action. Several participants noted that the need to stop was a legal requirement, with some explicitly noting that signs are placed at "dangerous" RLXs.

Compliance in the current study was higher than in field studies (12-41\%; Russell et al., 2007; Tey et al., 2011), but comparable to simulator studies (60-74\%; Lenné et al., 2011; Tey et al., 2013). Three factors can explain the relatively high compliance rates in simulator and on-road studies compared with field observations. The first is volunteer bias: participants in instrumented vehicle and simulator studies may be more conscientious and safety-conscious. Second, participants know their behaviour is being observed and recorded. Finally, Australian road rules dictate that stop signs can only be placed at RLXs with restricted sight distance, and in the current study some drivers noted that stop-controlled RLXs are a special class that warrant more cautious behaviour than other passive RLXs. This suggests that compliance with stop controls increases when they are used judiciously and drivers are aware of (and agree with) the rationale for their installation.

\subsection{Non-compliant drivers}

Instances of non-compliance can be examined in terms of both preceding factors (e.g., speed, which did not predict non-compliance) and the resulting consequences. Both unintentional and intentional non-compliance were associated with less extensive visual checks for trains, which is concerning given the restricted sight distance at these crossings. Non-compliant drivers also executed their visual checks farther back from the RLX, at which point they may not have had adequate visibility down the tracks to detect an approaching train, given the restricted visibility at all three RLXs. In addition, previous research has found train horns are significantly less audible in moving compared to idling vehicles (Dolan and Rainey, 2005), which implies that failing to stop also will impair drivers' ability to audibly detect trains. Analysis of approach speeds indicated that $100 \mathrm{~m}$ from the RLX, travel speeds were similar for compliant and non-compliant drivers; however, non-compliant drivers showed substantially less speed reduction, resulting in them proceeding through the stop sign at speeds of up to $56 \mathrm{~km} / \mathrm{h}$.

Drivers who reported unintentional non-compliance stated that they failed to detect the stop sign because they were focusing on other aspects of the environment. These situations all occurred at RLX 1, which several drivers considered demanding because it was at the end of a gravel road, immediately before a T-intersection. It was the only RLX that had a white crossbuck against a red background (see Figure 2), which potentially made the red stop sign relatively less salient. It would be worthwhile conducting more comprehensive field trials of sign salience and attentional capture, to 
ensure signs are easily detectable. However, of the drivers interviewed about unintentional noncompliance, only one indicated that they would have stopped if they had noticed the sign. This suggests that although improving sign salience will reduce unintentional non-compliance, some drivers might commit intentional violations instead.

Intentional violators acknowledged the stop sign but made a conscious decision not to stop based on their assessment of the situation. Two factors influenced this assessment. First, they believed that they had adequately searched for oncoming trains; this belief is erroneous as stop-controlled RLXs in Australia have inadequate sight distance. Second, they reported extensive experiences of driving across similar RLXs without seeing a train. This is consistent with previous research whereby drivers suggested the appropriate response at a stop-controlled RLX is to "slow down" and/or "look for trains" (Rudin-Brown et al., 2012; Tey et al., 2013). Observational research has found only one-third of drivers make adequate visual checks at low-frequency passive crossings (Wigglesworth, 2001), suggesting drivers become complacent when they know trains are rare. Focus group research has also found young drivers underestimate the risks of failing to scan at RLXs or failing to reduce speed, and do not expect to encounter trains at passive RLXs (Davey et al., 2008).

\subsection{Implications}

The findings suggest non-compliance at stop-controlled RLXs can involve schema-based errors. All participants had repeated, regular exposure to RLXs, as the local area had a very high density of RLXs; however, most did not regularly drive through passive RLXs and infrequently encountered trains. Further, some novice drivers had never encountered a train at an RLX, despite having over 12 months' solo driving experience, and being required to have 120 hours of supervised practice before obtaining their licence. It appears some drivers possess schema that they will (almost) never encounter a train at a rural RLX (because they never have before), which leads them to underestimate risks. For example, they do not realise that they have restricted sight distance, as having never encountered a train previously they have never had an opportunity to assess the adequacy of sight distance on approach to an RLX. Furthermore, some drivers failed to detect the stop sign, because it is not a cue that they expect to encounter at RLXs. Some compliant drivers also reported having relevant schemata for stop-controlled RLXs (i.e., that the stop sign means it is "dangerous"); however, other compliant drivers appeared to employ basic rule-based decision-making (i.e., "stop at stop signs"). The diversity of responses observed suggests that a "one size fits all" approach to RLX safety is likely to fail, as it cannot accommodate the variety of decision-making strategies that different users employ.

The current findings have important implications for RLX design and safety. The fact that several participants reported failing to detect stop signs suggests that more salient signage and/or earlier presentation of signage may be required. The findings also raise a larger question around whether stop 
signs at RLXs are necessary or appropriate. Although some campaigns explicitly note that stopcontrolled crossings have limited sight distance (CARRS-Q, 2015), the current study suggests many drivers remain ignorant of the rationale for installing stop signs. Alternative design solutions may be possible that address some of the key risks observed, namely: some drivers fail to detect the stop sign; some drivers detect the stop sign but do not realise that it signals an "unsafe" RLX with restricted sight distance; and some drivers detect the stop sign but ignore it because they do not expect to encounter a train. Redesign opportunities at the RLXs could be coupled with education campaigns highlighting the challenges drivers face at rural RLXs, particularly the fact that trains can be masked by vegetation on approach to RLXs, that train schedules can change or be unpredictable, and the importance of checking for trains at passive RLXs.

Our findings imply that, although substantial slowing is necessary to give drivers time to visually check for trains at passive RLXs, it may not be necessary to stop completely when no train is approaching. Drivers who made rolling stops and those who stopped completely spent the same amount of time visually checking for trains, and showed similar situation awareness. Stopping completely may be problematic for some vehicles, such as heavy vehicles, which lose momentum and require considerable time to regain speed and clear the RLX. Moreover, as noted in the introduction, crash analyses have yielded conflicting results regarding the safety performance of stop-controlled RLXs (Austin and Carson, 2002; McCollister and Pflaum, 2007; Millegan et al., 2009; Raub, 2009; Saccomanno et al., 2007; Yan et al., 2010a, 2010b). Furthermore, previous researchers have argued against using stop signs at RLXs due to the high rates of non-compliance observed, due to concerns this may generalise this behaviour and become non-compliant at stop-controlled highway and road intersections (Austin and Carson, Lerner et al., 2002; Raub, 2009). In our sample, several drivers reported generalising from their experience with road intersections to make their stop-or-go decision at the RLX, so it seems plausible that some drivers could generalise experience from RLXs back to intersections. Given that the underlying aim of placing a stop sign at an RLX is to provide drivers with more time to check for trains and increase safe decision-making, it would be worth focusing future RLX design efforts on interventions that can achieve these aims without requiring drivers to stop unnecessarily. These may involve traffic calming and speed control measures, coupled with other measures that make it easier to detect approaching trains (e.g., improving sight distance, where feasible) and encourage greater visual scanning.

\subsection{Limitations and future directions}

Although the current study adds to the literature on stop-controlled RLXs, it has limitations that should be noted. Most problematic is the fact that none of our participants encountered trains at any of the passive RLXs. This to a large extent reflects the usual experience of users of rural RLXs, as noted by 
our participants; our novice drivers had over 12 months' solo driving experience and drove across RLXs frequently, but most of them had never encountered a train when negotiating a RLX. Experienced drivers were more aware of the potential for encountering a train, but several of them mentioned that trains are infrequent. This represents a practical limitation of studying rural RLXs in naturalistic settings: it is relatively rare for road users to encounter a train at a rural RLX, so it is hard to study their responses without artificially manipulating the situation (e.g., using a closed track, with a train that comes when the participant is approaching). For this reason, several previous studies have used simulation as a means to explore drivers' responses to trains in rural environments (e.g., Lenné et al., 2011; RudinBrown et al., 2012; Tey et al., 2013).

Another limitation is that the current sample size, for both participants and RLXs, was relatively small. Notably, the sample of compliant drivers was much larger than the sample of violators, and there was relatively high consistency in both behaviour and self-reported cognitions among compliant drivers. In contrast, drivers who violated the stop controls reported more diverse motivations and cognitions. Thus although our findings reveal some drivers' reasons for committing violations at stop-controlled RLXs, caution is urged when generalising these results (especially the proportion of drivers reporting each factor) to the broader population. One option for future research would be to conduct a long-term self-report study of road users who commonly encounter passive RLXs; for instance, asking drivers to keep a diary record of how many RLXs they encounter each day and the events that occur. We previously used a similar method to explore how road users make stop-go decisions at RLXs, with the diary questions structured around CDM prompts (Beanland et al., 2016; Mulvihill et al., 2016). However, participants only completed the CDM if they saw a train on approach to the RLX, so the data yielded very few encounters with passive crossings. Future research could expand on this by having rural road users complete CDM diaries for all passive RLX encounters, regardless of train presence, to gain deeper understanding of the factors that influence their decision-making.

\section{Conclusions}

The current study explored factors associated with drivers' compliance or non-compliance with stop signs at rural RLXs. Most participants exhibited compliant behaviour, which they described as an automatic, rule-oriented response. In contrast, drivers who violated the stop sign either failed to detect its presence, or saw the sign but disregarded it because they underestimated the risks of proceeding (i.e., over-estimated sight distance). These findings highlight avenues for improving safety at rural RLXs through avenues including infrastructure design and education and awareness campaigns. 


\section{Acknowledgements}

This research was supported by an Australian Research Council (ARC) Linkage Grant (LP100200387) to the University of the Sunshine Coast, Monash University, and the University of Southampton, in partnership with the Victorian Rail Track Corporation, Transport Safety Victoria, Public Transport Victoria, Transport Accident Commission, Roads Corporation (VicRoads) and V/Line Passenger Pty Ltd. VB is supported by an ARC Discovery Early Career Researcher Award (DE150100083) and PS is supported by an ARC Future Fellowship (FT140100681). We gratefully acknowledge the support of the project partners, community participants and staff of the VicRoads Northern Region Office. We also thank Nebojsa Tomasevic for technical assistance, and Russell Boag and Georgia Lawrasia for data coding. 


\section{References}

Austin, R.D., Carson, J.L. 2002. An alternative accident prediction model for highway-rail interfaces. Accid Anal Prev 34 (1), 31-42. http://dx.doi.org/10.1016/S00014575(00)00100-7

Australian Transport Safety Bureau (ATSB). 2008. Railway level crossing safety bulletin. Canberra, Australia: ATSB.

Beanland, V., Lenné, M.G., Salmon, P.M., Stanton, N.A. 2016. Variability in decision-making and critical cue use by different road users at rail level crossings. Ergonomics 59 (6), 754-766. http://dx.doi.org/10.1080/00140139.2015.1095356

Cairney, P., Gunatillake, T., Wigglesworth, E. 2002. Reducing collisions at passive railway level crossings in Australia. Sydney, Australia: Austroads.

CARRS-Q (Centre for Accident Research and Road Safety - Queensland). 2015. Railway level crossing safety. Kelvin Grove, Australia: CARRS-Q. Retrieved from: http://www.carrsq.qut.edu.au/publications/corporate/level_crossing_fs.pdf

Davey, J., Wallace, A., Stenson, N., Freeman, J. 2008. Young drivers at railway crossings: An exploration of risk perception and target behaviours for intervention. Int J Inj Contr Saf Promot 15 (2), 57-64. http://dx.doi.org/10.1080/17457300801972221

Dolan, T.G., Rainey, J.E. (2005). Audibility of train horns in passenger vehicles. Hum Factors 47 (3), 613-629. http://dx.doi.org/10.1518/001872005774859999

Eluru, N., Bagheri, M., Miranda-Moreno, L.F., Fu, L. 2012. A latent class modeling approach for identifying vehicle driver injury severity factors at highway-railway crossings. Accid Anal Prev 47, 119-127. http://dx.doi.org/10.1016/j.aap.2012.01.027

Evans, A.W. 2011. Fatal accidents at railway level crossings in Great Britain 1946-2009. Accid Anal Prev 43 (5), 1837-1845. http://dx.doi.org/10.1016/j.aap.2011.04.019

Hoffman, R.R., Crandall, B., Shadbolt, N. 1998. Use of the critical decision method to elicit expert knowledge: A case study in the methodology of cognitive task analysis. Hum Factors 40 (2), 254-276.

Kasalica, S., Vukadinović, R., Lučanin, V. 2012. Study of drivers' behaviour at a passive railway crossing. Promet Traffic Transp 24 (3), 193-201. http://dx.doi.org/10.7307/ptt.v24i3.312

Klein, G.A., Calderwood, R., MacGregor, D. 1989. Critical decision method for eliciting knowledge. IEEE Trans Syst Man Cybern Syst 19 (3), 462-472. http://dx.doi.org/10.1109/21.31053

Lenné, M.G., Beanland, V., Salmon, P.M., Filtness, A.J., Stanton, N.A. 2013. Checking for trains: An on-road study of what drivers actually do at level crossings. In: N. Dadashi, A. Scott, J. R. Wilson, A. Mills (Eds.), Rail Human Factors: Supporting reliability, safety and cost reduction (pp. 53-59). London, UK: Taylor \& Francis. 
Lenné, M.G., Rudin-Brown, C.M., Navarro, J., Edquist, J., Trotter, M., Tomasevic, N. 2011. Driver behaviour at rail level crossings: Responses to flashing lights, traffic signals and stop signs in simulated rural driving. Appl Ergon 42 (4), 548-554. http://dx.doi.org/10.1016/j.apergo.2010.08.011

Lenné, M.G., Salmon, P.M., Beanland, V., Stanton, N.A., Filtness, A. 2013. On-road driving studies to understand why drivers behave as they do at regional rail level crossings. In: Proceedings of the 2013 Australasian Road Safety Research, Policing \& Education Conference, Canberra, Australia, pp. 1-7.

Lerner, N.D., Llaneras, R.E., McGee, H.W., Stephens, D.E., 2002. Traffic-Control Devices for Passive Railroad-Highway Grade Crossings. NCHRP Report 470. Washington, DC: Transportation Research Board.

Liu, J., Bartnik, B., Richards, S.H., Khattak, A.J. 2016. Driver behavior at highway-rail grade crossings with passive traffic controls: A driving simulator study. J Transp Saf Secur 8 (sup 1), 37-55. http://dx.doi.org/10.1080/19439962.2015.1043478

McCollister, G.M., Pflaum, C.C. 2007. A model to predict the probability of highway rail crossing accidents. Proc Inst Mech Eng F J Rail Rapid Trans 221 (3), 321-329. http://dx.doi.org/10.1243/09544097jrrt84

Millegan, H., Yan, X., Richards, S., Han, L. 2009. Evaluation of effectiveness of Stop sign treatments at highway-railroad grade crossings. Transp Res Rec 2122, 78-85. http://dx.doi.org/10.3141/2122-10

Mulvihill, C.M., Salmon, P.M., Beanland, V., Lenné, M.G., Read, G.J.M., Walker, G.H., Stanton, N.A. 2016. Using the decision ladder to understand road user decision making at actively controlled rail level crossings. Appl Ergon 56, 1-10. http://dx.doi.org/10.1016/j.apergo.2016.02.013

Neisser, U. 1976. Cognition and Reality: Principles and Implications of Cognitive Psychology. San Francisco, CA: Freeman.

Norman, D.A. 1981. Categorization of action slips. Psych Rev 88 (1), 1-15. http://dx.doi.org/10.1037/0033-295X.88.1.1

Plant, K.L., Stanton, N.A. 2013. The explanatory power of Schema Theory: theoretical foundations and future applications in Ergonomics. Ergonomics 56 (1), 1-15. http://dx.doi.org/10.1080/00140139.2012.736542

Plant, K.L., Stanton, N.A. 2013b. What is on your mind? Using the perceptual cycle model and critical decision method to understand the decision-making process in the cockpit. Ergonomics 56 (8), 1232-1250. http://dx.doi.org/10.1080/00140139.2013.809480

Pyrgidis, C., Papacharitou, E., Eleftheriadis, A. 2016. Risk management at railroad grade crossings: Proposal for a decision support system. Transp Res Procedia 14, 1394-1402. http://dx.doi.org/10.1016/j.trpro.2016.05.212 
Raub, R. 2009. Examination of highway-rail grade crossing collisions nationally from 1998 to 2007. Transp Res Rec 2122, 63-71. http://dx.doi.org/10.3141/2122-08

Read, G.J.M., Beanland, V., Lenné, M.G., Stanton, N.A., Salmon, P.M. 2017. Integrating Human Factors Methods and Systems Thinking for Transport Analysis and Design. CRC Press.

Reason, J.T., Manstead, A., Stradling, S., Baxter, J.S., Campbell, K. 1990. Errors and violations on the roads: A real distinction? Ergonomics 33 (10-11), 1315-1332. http://dx.doi.org/10.1080/00140139008925335

Rudin-Brown, C. M., Lenné, M.G., Edquist, J., Navarro, J. 2012. Effectiveness of traffic light vs. boom barrier controls at road-rail level crossings: A simulator study. Accid Anal Prev 45, 187-194. http://dx.doi.org/10.1016/j.aap.2011.06.019

Russell, E.R., Shah, H.D., Rys, M.J. 2007. Study of drivers' behavior at passive railroad-highway grade crossings. Fayetteville, AR: Mack-Blackwell Transportation Center.

Saccomanno, F.F., Park, P.Y.-J., Fu, L. 2007. Estimating countermeasure effects for reducing collisions at highway-railway grade crossings. Accid Anal Prev 39 (2), 406-416. http://dx.doi.org/10.1016/j.aap.2006.08.016

Salmon, P.M., Beanland, V., Filtness, A.J., Lenné, M.G., Stanton, N. 2013a. Waiting for warning: Driver situation awareness at rural rail level crossings. In M. Anderson (Ed.), Contemporary Ergonomics \& Human Factors 2013. (pp. 403-410). Cambridge, UK: Taylor \& Francis.

Salmon, P. M., Goode, N.A., Spiertz, A., Thomas, M., Grant, E., Clacy, A. 2017. Is it really good to talk? Testing the impact of providing concurrent verbal protocols on driving performance. Ergonomics 60 (6), 770-779. http://dx.doi.org/10.1080/00140139.2016.1214752

Salmon, P., Lenné, M., Beanland, V., Young, K., Filtness, A., Stanton, N., Read, G. 2014a. From the Bush to the Burbs: a comparison of driver situation awareness at rural and urban railway level crossings. In: Proceedings of the 2014 Australasian Road Safety Research, Policing \& Education Conference, Canberra, Australia, pp. 1-10.

Salmon, P.M., Lenné, M.G., Walker, G.H., Stanton, N.A., Filtness, A. 2014b. Exploring schemadriven differences in situation awareness between road users: an on-road study of driver, cyclist and motorcyclist situation awareness. Ergonomics 57 (2), 191-209. http://dx.doi.org/10.1080/00140139.2013.867077

Salmon, P.M., Read, G.J.M., Stanton, N.A., Lenné, M.G. 2013b. The crash at Kerang: Investigating systemic and psychological factors leading to unintentional non-compliance at rail level crossings. Accid Anal Prev 50, 1278-1288. http://dx.doi.org/10.1016/j.aap.2012.09.029

Standards Australia. 2007. Manual of uniform traffic control devices. Part 7: Railway crossings (AS 1742.7--2007). Sydney, Australia: Standards Australia. 
Stanton, N.A., Salmon, P.M., Walker, G.H., Jenkins, D. 2009. Genotype and phenotype schemata and their role in distributed situation awareness in collaborative systems. Theor Issues Ergon Sci 10 (1), 43-68. http://dx.doi.org/10.1080/14639220802045199

Stanton, N.A., Walker, G.H., Young, M.S., Kazi, T., Salmon, P.M. 2007. Changing drivers' minds: the evaluation of an advanced driver coaching system. Ergonomics 50 (8), 1209-1234. http://dx.doi.org/10.1080/00140130701322592

Tey, L.-S., Ferreira, L., Wallace, A. 2011. Measuring driver responses at railway level crossings. Accid Anal Prev 43 (6), 2134-2141. http://dx.doi.org/10.1016/j.aap.2011.06.003

Tey, L.-S., Wallis, G., Cloete, S., Ferreira, L., Zhu, S. 2013. Evaluating driver behavior toward innovative warning devices at railway level crossings using a driving simulator. J Transp Saf Secur 5 (2), 118-130. http://dx.doi.org/10.1080/19439962.2012.731028

Walker, G.H., Stanton, N.A., Kazi, T.A., Salmon, P.M., Jenkins, D.P. 2009. Does advanced driver training improve situational awareness? Appl Ergon 40(4), 678-687. http://dx.doi.org/10.1016/j.apergo.2008.06.002

Ward, N., Wilde, G. 1996. Driver approach behaviour at an unprotected railway crossing before and after enhancement of lateral sight distances: An experimental investigation of risk perception and behavioural compensation hypothesis. Saf Sci 22(1-3), 63-75.

Wigglesworth, E. 2001. A human factors commentary on innovations at railroad-highway grade crossings in Australia. J Saf Res 32 (3), 309-321.

Yan, X., Han, L.D., Richards, S., Millegan, H. 2010a. Train-vehicle crash risk comparison between before and after stop signs installed at highway-rail grade crossings. Traffic Inj Prev 11 (5), 535-542. http://dx.doi.org/10.1080/15389588.2010.494314

Yan, X., Richards, S., Su, X. 2010b. Using hierarchical tree-based regression model to predict train-vehicle crashes at passive highway-rail grade crossings. Accid Anal Prev 42 (1), 6474. http://dx.doi.org/10.1016/j.aap.2009.07.003

Yeh, M., Multer, J. 2007. Traffic control devices and barrier systems at grade crossings: Literature review. Transp Res Rec 2030, 69-75. http://dx.doi.org/10.3141/2030-10

Young, K.L., Lenné, M.G., Beanland, V., Salmon, P.M., Stanton, N.A. 2015. Where do novice and experienced drivers direct their attention on approach to urban rail level crossings? Accid Anal Prev 77, 1-11. http://dx.doi.org/10.1016/j.aap.2015.01.017 